\title{
Sensitivity Analysis of a Bayesian Network for Reasoning about Digital Forensic Evidence
}

\author{
Richard E. Overill, Jantje A. M. Silomon \\ Department of Computer Science, King's College London, \\ Strand, London WC2R 2 LS \\ \{richard.overill, jantje.a.silomon\}@kcl.ac.uk
}

\author{
Michael Y.K. Kwan, Kam-Pui Chow, Frank Y.W. Law, Pierre \\ K.Y. Lai \\ Department of Computer Science, University of Hong Kong, \\ Pokfulam Road, Hong Kong \\ \{ykkwan, chow, ywlaw, kylai\}@cs.hku.hk
}

\begin{abstract}
A Bayesian network representing an actual prosecuted case of illegal file sharing over a peer-to-peer network has been subjected to a systematic and rigorous sensitivity analysis. Our results demonstrate that such networks are usefully insensitive both to the occurrence of missing evidential traces and to the choice of conditional evidential probabilities. The importance of this finding for the investigation of digital forensic hypotheses is highlighted.
\end{abstract}

Keywords-Bayesian network; digital forensic investigation; digital evidence; sensitivity analysis.

\section{INTRODUCTION AND BACKGROUND}

Bayesian Belief Networks (BBNs) [1] have recently been applied to reasoning about available evidence in digital forensic investigations [2]. BBNs are used to quantify the evidential strengths of investigative hypotheses and hence enhance the reliability and traceability of the results produced by digital forensic investigations.

In [2], the BBN for a Hong Kong court case involving the use of a BitTorrent (BT) peer-to-peer (P2P) network to act as the initial 'seeder' for illegally uploading a copyright protected audio-visual file for subsequent distribution was constructed and examined. In Hong Kong only the uploading of copyright protected material is prohibited whereas in the UK any activity infringing copyright is forbidden. It was found that the BBN is a useful tool for quantifying and propagating the strengths of investigative hypotheses and their supporting evidence. However, there is an inherent subjectivity involved in assigning conditional probabilities to posterior evidence in the BBN.
This was alleviated to some extent through the use of a survey of expert digital forensic investigators and aggregating their responses.

In addition to the potential subjectivity of the conditional evidential probabilities, also known as likelihoods, a second issue to be addressed is that of missing evidence. It is often the case that not all of the expected evidential traces are actually recovered during a digital forensic investigation, and it is important to know the impact of one or more missing evidential traces upon the overall strength of the hypothesis under investigation.

In this paper, we address both of these issues by performing a rigorous sensitivity analysis on the BT BBN from [2], involving the systematic removal of evidential traces from this BBN and the systematic replacement of the aggregated likelihoods by their respective minimum and maximum values.

We detail the methodology used in section 2 below. In section 3 we discuss the results of the sensitivity analysis and in section 4 we present our conclusions.

\section{SEnSitivity AnAlysis Methodology}

As in [2], all BT BBN calculations were performed with the Microsoft MSBNx Editor [3].

\section{A. Missing evidential traces}

The structure of the BT BBN from [2] is given in Figure 1. It will be noted that this BBN contains a root hypothesis $(\mathrm{H})$, five sub-hypothesis $\left(\mathrm{H}_{1}-\mathrm{H}_{5}\right)$ and 18 associated evidential traces $\left(\mathrm{E}_{1}-\mathrm{E}_{18}\right)$. It is the combination of these traces, rather than any individual subset, that is 
required to formulate the prosecution's case. We have systematically removed the evidential traces according to the following schema:

1) each individual trace (Table A1);

2) all possible pairs of traces (Table A2);

3 ) a sample of $n$-tuples of traces; $3 \leq n \leq 9$ (Table A3).

\section{B. Minimum and maximum evidential likelihoods}

We have systematically replaced the aggregated likelihoods from the original survey [2] by the minimum and maximum values of the responses provided by the sample of 31 expert digital forensic investigators. We have removed 'outlier' values from this sample by discounting any single response lying at either extreme of the range.

The results of this study are given in Table A4. In addition, we have simultaneously set all the likelihoods to their respective minimum and maximum values in turn. The outcome of these tests can be found at the foot of Table A4. The result obtained using the original aggregated values, as reported in [2], is given at the head of Table A1 for purposes of comparison.

\section{DISCUSSION OF RESULTS}

The results of our sensitivity analysis are collected in the Tables in the Appendix.

\section{A. Missing evidential traces}

Table A1 demonstrates that the output of the BT BBN is remarkably stable towards any single missing evidential trace. The mean percentage deviation of these values from the case where no traces are missing is $-0.8 \%$. Similarly, the results in Table A2 show that any pair of missing evidential traces does not greatly impact the probability of the BT BBN hypothesis. Here the mean percentage deviation is $-2.0 \%$. These deviations are negative since the absence of a trace decreases the probability of the root hypothesis. It will however be noted that the entries in Tables A1 and A2 involving $E_{1}, E_{2}$ and $E_{3}$ (belonging to $H_{1}$ ) as well as $\mathrm{E}_{13}$ and $\mathrm{E}_{18}$ (belonging to $\mathrm{H}_{5}$ ) are somewhat smaller in magnitude than the remainder. This can be attributed to the fact that, relative to the other hypotheses, $\mathrm{H}_{1}$ and $\mathrm{H}_{5}$ have fewer associated evidential traces and therefore the absence of any of these evidential traces has a proportionately greater impact on the BT BBN.

Even when more than two evidential traces are not recovered, the data in Table A3 suggests that in many cases the BT BBN can still yield a reasonably high probability from a prosecutorial perspective. Indeed, we have recorded (Table $\mathrm{A} 3$, penultimate line) one extreme case in which 7 of the 18 evidential traces are missing but the BT BBN still maintains a probability in excess of 0.8 .

\section{B. Minimum and maximum evidential likelihoods}

In Table A4 it will be seen that the BT BBN is also remarkably stable towards variation of the values of individual likelihoods: for the individual minimum and maximum values of the likelihoods the mean percentage deviations are $-0.2 \%$ and $+0.05 \%$ respectively. However, when all likelihoods are set to their respective minimum values simultaneously, the BT BBN experiences a much larger downward probability shift from the aggregated values $(-25.5 \%)$ than the corresponding upward shift when all likelihoods are set to their respective maximum values simultaneously $(+0.7 \%)$. This phenomenon can be explained by consulting the histogram of responses to the original survey [2], which exhibits a pronounced skewness towards the higher end of the range, due to the level of expert knowledge of the great majority of the respondents.

\section{SUMMARY AND CONCLUSIONS}

The sensitivity analysis reported in this paper demonstrates that the BT BBN used in [2] is insensitive to the occurrence of missing evidence and also to the choice of evidential likelihoods to an unexpected degree.

However, as would be anticipated, in very extreme cases of missing evidential traces (Table A3), or improbable choices of values for the likelihoods (Table A4, final line), much lower probabilities for the investigatory hypothesis are found.

Our overall finding is gratifying because it implies that the exact choice of values for the inherently subjective evidential likelihoods is not as critical as might have been expected. Values falling within the consensus of experienced expert investigators are sufficiently reliable to be used in the BBN model. Furthermore, our results imply that the inability to recover one or more evidential traces in a digital forensic investigation is not generally critical for the probability of the investigatory hypothesis under consideration.

Since the actual BT BBN used in this study is typical in form and structure of the BBNs employed to investigate other digital crime hypotheses (for example, the Internet auction fraud investigations [4]), this study offers reassurance that those BBNs will also exhibit similarly stable behaviour towards the non-recovery of evidential traces and the values chosen for the evidential likelihoods. 


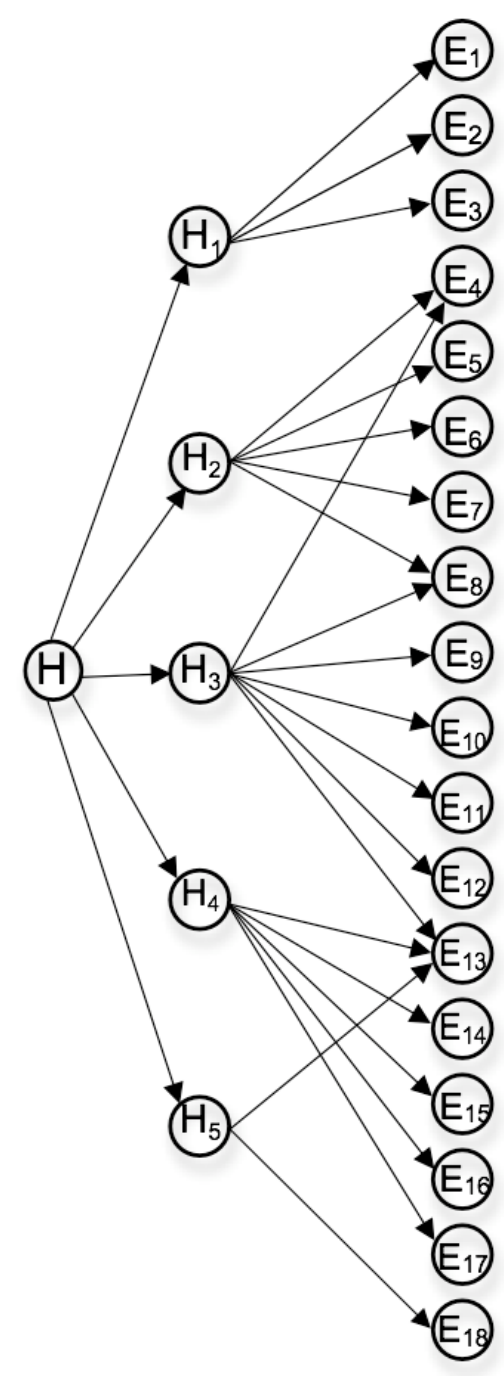

HYPOTHESES:

H The seized computer was used as the initial seeder to share the pirated file on a BitTorrent network

$\mathbf{H}_{\mathbf{1}}$ The pirated file was copied from the seized optical disk to the seized computer

$\mathbf{H}_{2}$ A torrent file was created from the copied file

$\mathbf{H}_{3}$ The torrent file was sent to newsgroups for publishing

$\mathbf{H}_{4}$ The torrent file was activated, which caused the seized computer to connect to the tracker server

$\mathbf{H}_{\mathbf{5}}$ The connection between the seized computer and the tracker server was maintained

\section{EVIDENCE:}

$\mathbf{E}_{\mathbf{1}}$ Modification time of the destination file equals that of the source file

$\mathbf{E}_{2}$ Creation time of the destination file is after its own modification time

$\mathbf{E}_{3}$ Hash value of the destination file matches that of the source file

$\mathbf{E}_{4}$ BitTorrent client software is installed on the seized computer

$\mathbf{E}_{5}$ File link for the shared file is created

$\mathbf{E}_{6}$ Shared file exists on the hard disk

$\mathbf{E}_{7}$ Torrent file creation record is found

E$_{8}$ Torrent file exists on the hard disk

E9 Peer connection information is found

$\mathbf{E}_{\mathbf{1 0}}$ Tracker server login record is found

$\mathbf{E}_{\mathbf{1 1}}$ Torrent file activation time is corroborated by its MAC time and link file

$\mathbf{E}_{12}$ Internet history record about the publishing website is found

$\mathbf{E}_{13}$ Internet connection is available

$\mathbf{E}_{\mathbf{1 4}}$ Cookie of the publishing website is found

$\mathbf{E}_{15}$ URL of the publishing website is stored in the web browser

$\mathbf{E}_{16}$ Web browser software is available

$\mathbf{E}_{17}$ Internet cache record about the publishing of the torrent file is found

$\mathbf{E}_{18}$ Internet history record about the tracker server connection is found

Figure 1: The BT BBN [2] 


\section{REFERENCES}

[1] J. Keppens and J. Zeleznikow, "A model based reasoning approach for generating plausible crime scenarios from evidence", Proceedings of the Ninth International Conference on Artificial Intelligence and Law (ICAIL'03), Edinburgh, Scotland, 24-28 June 2003, New York: ACM, 2003 , pp. $51-59$.

[2] M. Kwan, K.-P. Chow, F. Law and P. Lai, "Reasoning about evidence using Bayesian networks", Chapter 12, Advances in Digital Forensics IV, I Ray and S Shenoi, Eds. Berlin: Springer, 2008, pp. 142 - 155.

[3] Microsoft Research, MSBNx: Bayesian Network Editor and Tool Kit, Microsoft Corporation, Redmond, Washington, USA, 2001.

http://www.research.microsoft.com/adapt/MSBNx

[4] M. Kwan, R. E. Overill, K.-P. Chow, J. A. M. Silomon, H. Tse, F. Law and P. Lai, "Internet auction fraud investigations", Proceedings of the Sixth Annual IFIP WG 11.9 International Conference on Digital Forensics, Hong Kong, 3-6 January 2010, Advances in Digital Forensics VI, S Shenoi, Ed. Berlin: Springer, 2010, in press.

\section{APPENDIX}

TABLE A1

Single Missing Traces

\begin{tabular}{|c|c|}
\hline Missing Evidence & BT BBN $p(\mathrm{H})$ \\
\hline & \\
none [2] & 0.9255 \\
$\mathrm{E}_{1}$ & 0.9158 \\
$\mathrm{E}_{2}$ & 0.9158 \\
$\mathrm{E}_{3}$ & 0.9109 \\
$\mathrm{E}_{4}$ & 0.9252 \\
$\mathrm{E}_{5}$ & 0.9253 \\
$\mathrm{E}_{6}$ & 0.9240 \\
$\mathrm{E}_{7}$ & 0.9254 \\
$\mathrm{E}_{8}$ & 0.9249 \\
$\mathrm{E}_{9}$ & 0.9248 \\
$\mathrm{E}_{10}$ & 0.9248 \\
$\mathrm{E}_{11}$ & 0.9239 \\
$\mathrm{E}_{12}$ & 0.9247 \\
$\mathrm{E}_{13}$ & 0.8990 \\
$\mathrm{E}_{14}$ & 0.9252 \\
$\mathrm{E}_{15}$ & 0.9251 \\
$\mathrm{E}_{16}$ & 0.9242 \\
$\mathrm{E}_{17}$ & 0.9251 \\
$\mathrm{E}_{18}$ & 0.8623 \\
& \\
\hline
\end{tabular}

TABLE A3

Multiple Missing Traces

\begin{tabular}{|l|c|}
\hline \multicolumn{1}{|c|}{ Missing Traces } & BT BBN $p(\mathrm{H})$ \\
\hline & \\
$\mathrm{E}_{4}, \mathrm{E}_{8}, \mathrm{E}_{13}$ & 0.8928 \\
$\mathrm{E}_{1}, \mathrm{E}_{4}, \mathrm{E}_{8}, \mathrm{E}_{13}$ & 0.8794 \\
$\mathrm{E}_{9}, \mathrm{E}_{10}, \mathrm{E}_{11}, \mathrm{E}_{12}, \mathrm{E}_{14}, \mathrm{E}_{15}$ & 0.6613 \\
$\mathrm{E}_{4}, \mathrm{E}_{6}, \mathrm{E}_{8}, \mathrm{E}_{9}, \mathrm{E}_{11}, \mathrm{E}_{15}, \mathrm{E}_{16}$ & 0.6994 \\
$\mathrm{E}_{1}, \mathrm{E}_{2}, \mathrm{E}_{5}, \mathrm{E}_{7}, \mathrm{E}_{8}, \mathrm{E}_{10}, \mathrm{E}_{14}$ & 0.8042 \\
$\mathrm{E}_{4}, \mathrm{E}_{6}, \mathrm{E}_{8}, \mathrm{E}_{9}, \mathrm{E}_{11}, \mathrm{E}_{13}, \mathrm{E}_{15}, \mathrm{E}_{16}$ & 0.4645 \\
\hline
\end{tabular}

TABLE A4

BT BBN using Minimum and Maximum Likelihoods

\begin{tabular}{|c|c|c|}
\hline Evidence & BT BBN $p(\mathrm{H}) \min$ & $\mathrm{BT} \mathrm{BBN} p(\mathrm{H}) \max$ \\
\hline $\mathrm{E}_{1}$ & 0.9244 & 0.9256 \\
\hline$E_{2}$ & 0.9250 & 0.9256 \\
\hline$E_{3}$ & 0.9249 & 0.9256 \\
\hline $\mathrm{E}_{4}$ & 0.9254 & 0.9255 \\
\hline $\mathrm{E}_{5}$ & 0.9255 & 0.9255 \\
\hline $\mathrm{E}_{6}$ & 0.9252 & 0.9258 \\
\hline $\mathrm{E}_{7}$ & 0.9255 & 0.9255 \\
\hline $\mathrm{E}_{8}$ & 0.9252 & 0.9255 \\
\hline $\mathrm{E}_{9}$ & 0.9255 & 0.9256 \\
\hline $\mathrm{E}_{10}$ & 0.9255 & 0.9256 \\
\hline $\mathrm{E}_{11}$ & 0.9255 & 0.9256 \\
\hline $\mathrm{E}_{12}$ & 0.9254 & 0.9256 \\
\hline $\mathrm{E}_{13}$ & 0.9236 & 0.9255 \\
\hline $\mathrm{E}_{14}$ & 0.9253 & 0.9255 \\
\hline $\mathrm{E}_{15}$ & 0.9254 & 0.9255 \\
\hline $\mathrm{E}_{16}$ & 0.9254 & 0.9255 \\
\hline $\mathrm{E}_{17}$ & 0.9253 & 0.9255 \\
\hline $\mathrm{E}_{18}$ & 0.9012 & 0.9320 \\
\hline $\mathrm{E}_{1}-\mathrm{E}_{18}$ & 0.6896 & 0.9316 \\
\hline
\end{tabular}




\begin{tabular}{|c|c|c|c|c|c|c|c|c|c|c|c|c|c|c|c|c|c|c|}
\hline \multirow{18}{*}{ 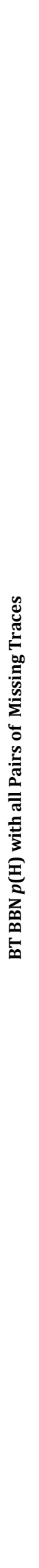 } & & & & & & & & & & & & & & & & & $\begin{array}{l}\overrightarrow{\mathrm{o}} \\
\infty \\
0 \\
0\end{array}$ & $\overrightarrow{~ ज े ~}$ \\
\hline & & & & & & & & & & & & & & & & $\begin{array}{l}\text { ơ } \\
\text { के } \\
\circ \\
0\end{array}$ & $\begin{array}{l}0 \\
\stackrel{0}{0} \\
\infty \\
0 \\
0\end{array}$ & ثै \\
\hline & & & & & & & & & & & & & & & 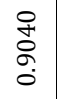 & 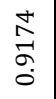 & $\mid \begin{array}{c}\overrightarrow{\overrightarrow{0}} \\
\infty \\
0 \\
0\end{array}$ & $\stackrel{n g}{9}$ \\
\hline & & & & & & & & & & & & & & $\begin{array}{l}\vec{\sigma} \\
\vec{\sigma} \\
\sigma\end{array}$ & $\begin{array}{l}\vec{\infty} \\
\stackrel{2}{ } \\
\stackrel{0}{0}\end{array}$ & $\begin{array}{l}\vec{\sigma} \\
\vec{\sigma} \\
\sigma\end{array}$ & $\begin{array}{c}0 \\
\mathbb{N} \\
\infty \\
0 \\
0\end{array}$ & ثै \\
\hline & & & & & & & & & & & & & $\begin{array}{l}0 \\
\text { do } \\
\infty \\
0 \\
0\end{array}$ & $\begin{array}{l}\text { Oొ } \\
\infty \\
\infty \\
0 \\
0\end{array}$ & 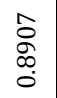 & $\begin{array}{l}0 \\
\mathscr{0} \\
\infty \\
0 \\
0\end{array}$ & \begin{tabular}{|c}
$\infty$ \\
$\vdots$ \\
$\vdots$ \\
$\infty$ \\
0 \\
0
\end{tabular} & $\stackrel{m}{*}$ \\
\hline & & & & & & & & & & & & $\begin{array}{l}\text { oे } \\
0 \\
\infty \\
0 \\
0\end{array}$ & 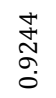 & 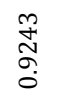 & 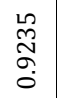 & $\begin{array}{l}\stackrel{3}{\sim} \\
\text { S. }\end{array}$ & 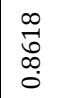 & 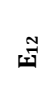 \\
\hline & & & & & & & & & & & $\begin{array}{l}1 \\
0 \\
0 \\
0 \\
0\end{array}$ & $\mid \begin{array}{l}0 \\
\infty \\
\infty \\
\infty \\
0 \\
0\end{array}$ & 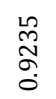 & $\begin{array}{l}\stackrel{L}{N} \\
\text { } \\
0 \\
0\end{array}$ & $\begin{array}{l}\stackrel{0}{\mathbf{N}} \\
\text { o. } \\
\stackrel{0}{0}\end{array}$ & 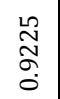 & $\begin{array}{c}\vec{F} \\
0 \\
\infty \\
0 \\
0\end{array}$ & Fi \\
\hline & & & & & & & & & & 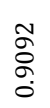 & $\begin{array}{l}0 \\
\hat{\sigma} \\
\sigma \\
0\end{array}$ & $\begin{array}{c}\text { Iै } \\
\text { ه } \\
0 \\
0\end{array}$ & 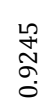 & \begin{tabular}{l}
\multirow{J}{*}{} \\
S. \\
0
\end{tabular} & 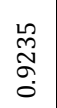 & 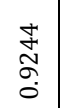 & 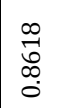 & जे \\
\hline & & & & & & & & & $\begin{array}{l}\stackrel{+}{\hat{H}} \\
\stackrel{\sigma}{0}\end{array}$ & $\begin{array}{l}\text { مे } \\
\text { ठे } \\
\text { o. }\end{array}$ & $\begin{array}{l}\stackrel{2}{1} \\
\text { aे } \\
0\end{array}$ & $\begin{array}{c}\text { If } \\
\text { of } \\
0 \\
0\end{array}$ & 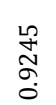 & $\begin{array}{l}\stackrel{+}{J} \\
\text { o. } \\
0\end{array}$ & 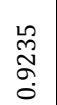 & 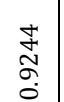 & $\begin{array}{l}\infty \\
\overrightarrow{1} \\
\infty \\
0\end{array}$ & 요 \\
\hline & & & & & & & & $\begin{array}{l}\overrightarrow{\tilde{N}} \\
\text { o. }\end{array}$ & $\begin{array}{l}\vec{S} \\
\text { o. } \\
0\end{array}$ & $\begin{array}{l}\text { fo } \\
\text { t. } \\
\text { o. }\end{array}$ & $\begin{array}{l}\infty \\
\stackrel{\infty}{\sigma} \\
\sigma \\
0\end{array}$ & $\begin{array}{c}N \\
N \\
\infty \\
\infty \\
0 \\
0\end{array}$ & $\begin{array}{l}\text { N } \\
\text { o. } \\
0\end{array}$ & $\begin{array}{l}\vec{N} \\
\text { o. } \\
0\end{array}$ & 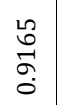 & \begin{tabular}{l}
$\vec{N}$ \\
\multirow{J}{O}{} \\
$\sigma$
\end{tabular} & 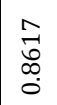 & 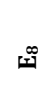 \\
\hline & & & & & & & $\begin{array}{l}\underset{J}{J} \\
\sigma \\
\delta\end{array}$ & $\begin{array}{l}\text { fे } \\
\text { S. } \\
\text { O }\end{array}$ & 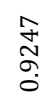 & $\begin{array}{l}\hat{\tilde{N}} \\
\text { o. } \\
0\end{array}$ & 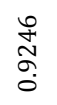 & $\begin{array}{l}\infty \\
\infty \\
\infty \\
\infty \\
0\end{array}$ & 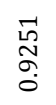 & $\begin{array}{l}\text { 오 } \\
\text { o. } \\
\text {. }\end{array}$ & $\begin{array}{l}\hat{\sigma} \\
\hat{\sigma} \\
0\end{array}$ & 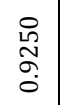 & $\begin{array}{c}\vec{N} \\
\mathbb{W} \\
\infty \\
0 \\
0\end{array}$ & s \\
\hline & & & & & & \begin{tabular}{l} 
兄 \\
\multirow{\sigma}{\sigma}{} \\
0
\end{tabular} & $\mid \begin{array}{l}\sigma \\
\sigma \\
\sigma \\
\sigma \\
\sigma\end{array}$ & 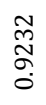 & 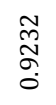 & 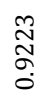 & 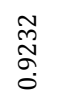 & $\begin{array}{l}\stackrel{0}{2} \\
\text { م. } \\
\infty \\
0 \\
0\end{array}$ & 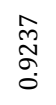 & $\begin{array}{l}\stackrel{\infty}{N} \\
\sigma \\
\stackrel{0}{0}\end{array}$ & 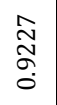 & \begin{tabular}{l}
0 \\
\multirow{N}{N}{} \\
$\sigma$ \\
0
\end{tabular} & $\begin{array}{c}\hat{\alpha} \\
\text { L } \\
\infty \\
0 \\
0\end{array}$ & نت:ن \\
\hline & & & & & $\begin{array}{l}\text { ळ. } \\
\stackrel{0}{\circ} \\
0\end{array}$ & $\begin{array}{l}\text { Oे } \\
\text { ूे } \\
0\end{array}$ & $\begin{array}{l}\text { O్ } \\
\text { o. } \\
0\end{array}$ & $\begin{array}{l}\text { I্ } \\
\text { Sू } \\
0\end{array}$ & $\begin{array}{l}\text { I } \\
\text { S. } \\
0\end{array}$ & $\begin{array}{l}\hat{\tilde{N}} \\
\text { s. } \\
0\end{array}$ & 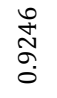 & $\begin{array}{l}\infty \\
\infty \\
\infty \\
\infty \\
0\end{array}$ & 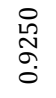 & 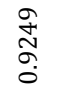 & 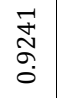 & 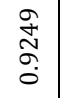 & $\begin{array}{c}0 \\
\mathbb{N} \\
\infty \\
0 \\
0\end{array}$ & 崮 \\
\hline & & & & $\begin{array}{l}\text { స్ } \\
\text { o. }\end{array}$ & $\mid \begin{array}{c}0 \\
0 \\
0 \\
0 \\
0 \\
0\end{array}$ & $\begin{array}{l}\text { ָे } \\
\text { S. } \\
0\end{array}$ & 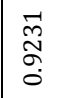 & 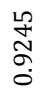 & 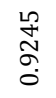 & 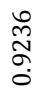 & 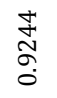 & $\begin{array}{l}\infty \\
\infty \\
\infty \\
\infty \\
0\end{array}$ & 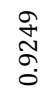 & 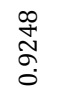 & $\begin{array}{l}\tilde{N} \\
\tilde{N} \\
\sigma\end{array}$ & 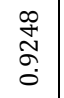 & $\begin{array}{l}\infty \\
\stackrel{\infty}{0} \\
\infty \\
0 \\
0\end{array}$ & ज्ञ \\
\hline & & & 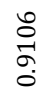 & $\begin{array}{l}\hat{o} \\
\text { ò } \\
\text { o. }\end{array}$ & $\begin{array}{l}\vec{a} \\
\text { S. } \\
\text { o. }\end{array}$ & $\begin{array}{l}\text { o } \\
\text { ă } \\
\text { o. }\end{array}$ & \begin{tabular}{|l} 
ô \\
$\sigma$ \\
$\sigma$ \\
0
\end{tabular} & $\begin{array}{l}\vec{\sigma} \\
0 \\
\sigma \\
0\end{array}$ & $\begin{array}{l}\overrightarrow{0} \\
0 \\
0 \\
0\end{array}$ & $\begin{array}{l}\text { Qे } \\
\text { Qे } \\
\text { Oे }\end{array}$ & $\begin{array}{l}\circ \\
\stackrel{0}{0} \\
0 \\
0\end{array}$ & 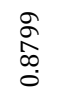 & $\begin{array}{l}\text { L } \\
0 \\
\sigma \\
0\end{array}$ & $\begin{array}{l}\text { 崩 } \\
\text { o. }\end{array}$ & 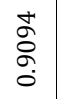 & $\begin{array}{l}\text { dे } \\
0 \\
\sigma \\
0\end{array}$ & $\begin{array}{c}n \\
0 \\
\infty \\
\infty \\
0\end{array}$ & 99 \\
\hline & & 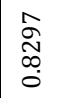 & $\begin{array}{l}\text { 吕 } \\
\text { م్ } \\
\sigma \\
0\end{array}$ & $\begin{array}{l}\text { مٌ } \\
\text { م్ } \\
\text { o. }\end{array}$ & 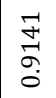 & $\begin{array}{l}\text { ñ } \\
\text { sू. } \\
\text { o. }\end{array}$ & $\begin{array}{l}\overrightarrow{5} \\
\text { مू } \\
0\end{array}$ & $\begin{array}{l}\text { مू } \\
\text { nू. } \\
\text { o. }\end{array}$ & $\begin{array}{l}0 \\
\text { nn } \\
\sigma \\
0\end{array}$ & $\begin{array}{l}\text { o } \\
\text { t. } \\
\text { o. }\end{array}$ & $\begin{array}{l}0 \\
\text { nn } \\
\sigma \\
0\end{array}$ & $\begin{array}{l}\text { B. } \\
\infty \\
\infty \\
0 \\
0\end{array}$ & $\begin{array}{l}\text { L } \\
\text { م⿱ } \\
\sigma \\
0\end{array}$ & 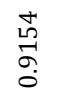 & 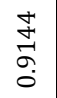 & $\begin{array}{l}\text { 曷 } \\
\text { o. }\end{array}$ & $\begin{array}{c}\hat{2} \\
\mathfrak{f} \\
\infty \\
0 \\
0\end{array}$ & 포 \\
\hline & $\begin{array}{c}\infty \\
\infty \\
\infty \\
\infty \\
0 \\
0\end{array}$ & $\begin{array}{c}\stackrel{\hat{~}}{\infty} \\
\text { o. } \\
0\end{array}$ & 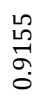 & $\begin{array}{l}\text { مै } \\
\text { h్ } \\
\text { o. }\end{array}$ & $\begin{array}{l}\vec{F} \\
\sigma \\
0\end{array}$ & $\begin{array}{l}\hat{n} \\
\text { nू } \\
\text { o. }\end{array}$ & 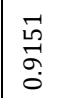 & 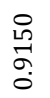 & $\begin{array}{l}0 \\
\text { hn } \\
\sigma \\
0\end{array}$ & $\begin{array}{l}\text { o } \\
\text { da } \\
\text { o. }\end{array}$ & $\begin{array}{l}\text { م⿱ } \\
\text { ha } \\
0\end{array}$ & $\begin{array}{l}\mathscr{8} \\
\infty \\
\infty \\
0 \\
0\end{array}$ & 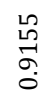 & $\begin{array}{l}\text { 曷 } \\
\sigma \\
\text { 。. }\end{array}$ & 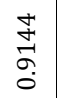 & $\begin{array}{l}\text { 曷 } \\
\text { ă }\end{array}$ & $\begin{array}{l}\hat{2} \\
\dot{f} \\
\infty \\
0 \\
0\end{array}$ & $\overrightarrow{4}$ \\
\hline & జ్ & 需 & + & 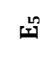 & దீ & is & ఉ & ஸి & ثิ & (I) & $\stackrel{\sim}{*}$ & $\stackrel{m}{\omega}$ & 志 & 总 & ثْتَ & $\stackrel{1}{1}$ & $\sqrt[\infty]{\sqrt{1}}$ & \\
\hline
\end{tabular}

